\title{
Future Prospects of 3D Human Chromosome Imaging by Serial Block Face Scanning Electron Microscopy \\ Mohammed Yusuf ${ }^{1,2 *}$ Bo Chen ${ }^{1,2}$ and lan Robinson ${ }^{1,2}$
}

${ }^{1}$ London Centre for Nanotechnology, University College London, London, UK

${ }^{2}$ Research Complex at Harwell, Rutherford Appleton Laboratory, Oxon, UK

Keywords: Chromosome; Serial block-face scanning electron microscopy; Three-dimensional imaging

\section{Commentary}

The higher order structure of human chromosomes remains to be elucidated with the $30 \mathrm{~nm}$ mystery still remaining. For internal structure determination, transmission electron microscopy (TEM) cannot be used as the chromosomes are too thick (approx. 1.4 microns) and scanning electron microscopy (SEM) is a surface imaging technique. For this purpose, the three-dimensional (3D) serial block face scanning electron microscopy (SBFSEM) was used on imaging mitotic human chromosome for the first time [1].

The SBFSEM technique [2] uses a diamond knife to cut thin slices of samples embedded into resin after preparing the samples using standard electron microscopy preparation techniques. The serial images by the SBFSEM do not need to be aligned because the system has automated image acquisition [2]. No published protocols were available for chromosome sample preparation for SBFSEM but rather for tissue samples [3] including rat liver to investigate chromatin in interphase nuclei [4]. This study used multiple fixation steps with complex longtime sample preparation procedures [4]. The chromosome sample preparation procedure for SBFSEM by Yusuf et al. [1] is much simpler and shorter. This study used centrifugation after each preparation step causing sample loss therefore the study successfully optimised the sample preparation procedures by starting with large scale cell cultures, synchronization and pooling samples together to get a concentrated starting yield. Further improvements were made after cleaning the chromosome sample by filtration, staining using platinum blue (a DNA specific heavy metal stain); [5] and embedding in a small amount of resin. Full 3D was obtained using the images taken and the typical $\mathrm{X}$ shaped chromosome was obtained with no internal structure. Further sample preparation changes from polyamine to methanol acetic acid showed internal pores [1].

SBFSEM has been used down to $20 \mathrm{~nm}$ sections showing clear signs of internal structure [1]. Even though porous features or cavities were seen on the chromosome arms, the resolution was limited to 11 $\times 11 \times 20 \mathrm{~nm}$. Charging effects and radiation damage were believed to give the current limit of resolution. Despite the $11 \mathrm{~nm}$ nominal lateral resolution, no $30 \mathrm{~nm}$ structures were seen. New images with $8.3 \times 8.3$ $\mathrm{nm}$ pixel size show consistent staining in each slice (Figure 1a) and in slices further down in the series (Figure 1b) of the same chromosome (150 nm apart), also having porous information. Complementary methods such as focused ion beam scanning electron microscopy (FIBSEM) have provided 3D information on plant chromosomes (not human) due to sample preparation difficulties [6]. 3D X-ray coherent diffraction imaging (CDI) provided $120 \mathrm{~nm}$ resolution with little internal fine structure on a human chromosome [7]. Therefore there is no doubt that the SBFSEM method will be further used in the future for exploring higher order structure of the human genome, hopefully providing higher resolution as the techniques are improving,
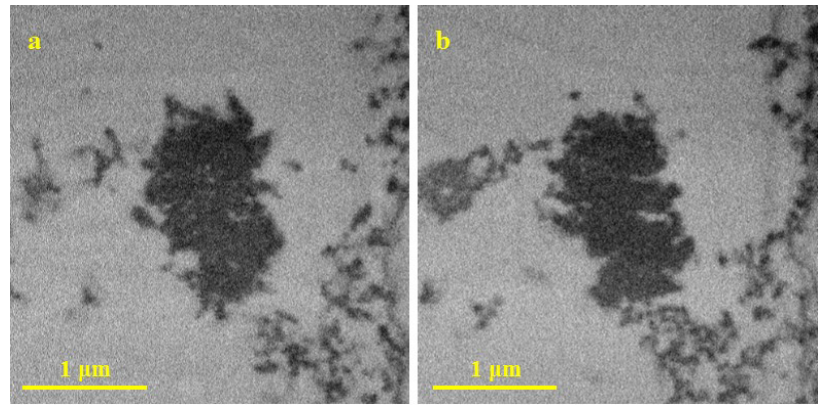

Figure 1: SBFSEM of a single human mitotic chromosome prepared from a lymphoblastoid cell line (GM18507). 2D sections are stained with platinum blue. (a) shows a section after SBFSEM from a whole chromosome b) is a section further down the series being $150 \mathrm{~nm}$ apart from (a). Pixel size is $8.3 \times 8.3 \mathrm{~nm}$.

for example employing new generation back scattered electron (BSE) detectors. Various sample preparation procedures need to be developed that would include decondensing the chromatin by removing divalent cations, elimination of hypotonic buffer and imaging chromosomes by directly slicing cells. Currently no cryogenic stage for the instrument is available therefore the samples have to be imaged at room temperature after embedding in resin, however freeze substitution [8] of chromosomes after high pressure freezing would be a positive way forward. This would reduce the damage rate by preventing the diffusion of free radicals. Cryo-FIB has been used for cell imaging [9] and is yet to be experimented on human chromosomes in a single cell.

\section{Acknowledgment}

This work was supported by the Biotechnology and Biological Sciences Research Council (BBSRC), grant BB/H022597/1. Also we would like to thank Professor George Thompson and Mr Teruo Hashimoto for preparing the resin sample and use of the SBFSEM microscope. This was done at the Corrosion \& Protection Centre, School of Materials, The University of Manchester, UK Therefore the Engineering and Physical Sciences Research Council (EPSRC) is also acknowledged for support of the LATEST2 Programme Grant and the associated imaging facilities.

\section{References}

1. Yusuf M, Chen B, Hashimato T, Estandarte AK, Thompson GE, et al. (2014) Staining and embedding of human chromosomes for 3D Serial Block Face Scanning Electron Microscopy. BioTechniques 57: 302-307.

*Corresponding author: Mohammed Yusuf, London Centre for Nanotechnology, University College London, London, Research Complex at Harwell, Rutherford Appleton Laboratory, Oxon, UK, Tel: +44 207679 2000; E-mail: yusuf.mohammed@ucl.ac.uk

Received March 14, 2016; Accepted April 05, 2016; Published April 07, 2016

Citation: Yusuf M, Chen B, Robinson I (2016) Future Prospects of 3D Human Chromosome Imaging by Serial Block Face Scanning Electron Microscopy. Single Cell Biol 5: 134. doi:10.4172/2168-9431.1000134

Copyright: ( 2016 Yusuf M, et al. This is an open-access article distributed under the terms of the Creative Commons Attribution License, which permits unrestricted use, distribution, and reproduction in any medium, provided the original author and source are credited. 
Citation: Yusuf M, Chen B, Robinson I (2016) Future Prospects of 3D Human Chromosome Imaging by Serial Block Face Scanning Electron Microscopy. Single Cell Biol 5: 134. doi:10.4172/2168-9431.1000134

Page 2 of 2

2. Denk W, Horstmann H (2004) Serial block-face scanning electron microscopy to reconstruct three-dimensional tissue nanostructure. PLoS Biol 2: e329.

3. Starborg T, Kalson NS, Lu Y, Mironov A, Cootes TF, et al. (2013) Using transmission electron microscopy and 3 View to determine collagen fibril size and three-dimensional organization. Nat Protoc 8: 1433-1448.

4. Rouquette J, Genoud C, Vazquez-Nin GH, Kraus B, Cremer T, et al. (2009) Revealing the high-resolution three-dimensional network of chromatin and interchromatin space: a novel electron-microscopic approach to reconstructing nuclear architecture. Chromosome Res 17: 801-810.

5. Wanner G, Formanek H (1995) Imaging of DNA in human and plant chromosomes by high-resolution scanning electron microscopy. Chromosome Res 3: 368-374.
6. Schroeder-Reiter E, Pérez-Willard F, Zeile U, Wanner G (2009) Focused ion beam (FIB) combined with high resolution scanning electron microscopy: a promising tool for 3D analysis of chromosome architecture. J Struct Biol 165: 97-106.

7. Nishino Y, Takahashi Y, Imamoto N, Ishikawa T, Maeshima K (2009) Threedimensional visualization of a human chromosome using coherent $\mathrm{X}$-ray diffraction. Phys Rev Lett 102: 018101

8. Webb R, Webb R (2015) Quick Freeze Substitution Processing of Biological Samples for Serial Block-face Scanning Electron Microscopy. Microscopy and Microanalysis, 21: 1115-1116.

9. Schertel A, Snaidero N, Han HM, Ruhwedel T, Laue M, et al. (2013) Cryo FIBSEM: volume imaging of cellular ultrastructure in native frozen specimens. $J$ Struct Biol 184: 355-360. 